\title{
Compact Microstrip Cross-Coupled Bandpass Filters Using Miniaturized Stepped Impedance Resonators
}

\author{
Chi-Feng Chen, Ting-Yi Huang, and Ruey-Beei Wu \\ Department of Electrical Engineering and Graduate Institute of Communication Engineering, National Taiwan University, Taipei
} 106, Taiwan, R.O.C.

\begin{abstract}
Novel compact microstrip cross-coupled bandpass filters realized by miniaturized stepped impedance resonators (SIR's) are proposed. The cross coupling between non-adjacent resonators is introduced to exhibit a single pair of transmission zeros near the passband at finite frequencies and thus much better selectivity. The optimal design for miniaturized stepped impedance resonator is employed and realized by several layouts in order to reduce the filter size. Two design examples of four-pole cross-coupled filters are described in this paper. The measured results are in good agreement with the simulated predictions. Each proposed cross-coupled filter not only has a compact size but also has high-selectivity and a very wide stopband response.
\end{abstract}

Index Terms - Cross-coupled filters, microstrip filters, stepped impedance resonators, transmission zeros.

\section{INTRODUCTION}

$\mathrm{RF} /$ microwave bandpass filters are essential circuits in the wireless communication systems, whose trend is towards compact size and high performance in recent years. Thus, the elliptic or quasi-elliptic filters realized by cross coupling were introduced in order to reduce the size and improve the selectivity of bandpass filters [1-8]. They may exhibit a single pair of transmission zeros near the passband due to a cross coupling, so that the possible interference in the stopband can be rejected. However, these filters often have some disadvantages: the circuit sizes are large and the stopband rejections are not good enough.

For traditional microstrip parallel-coupled half-wavelength resonator bandpass filters [9-10], the first spurious passband is centered at twice the mid-band frequency due to the unequal even and odd mode phase velocity. This resulted in a narrow stopband between the fundamental response and the first spurious response. Therefore, the stepped impedance resonator (SIR), introduced by [11-13], was presented not only to control the spurious-mode frequencies, but also to reduce the resonator size.

In this work, two compact microstrip cross-coupled bandpass filters with good stopband rejection are proposed. By introducing the optimal design for miniaturized quarter-wavelength SIR, the minimum filter size can be obtained. In addition, the proposed bandpass filters have a wider stopband as compared with the conventional coupled-line filters which exhibit a repeated passband at twice the center frequency.

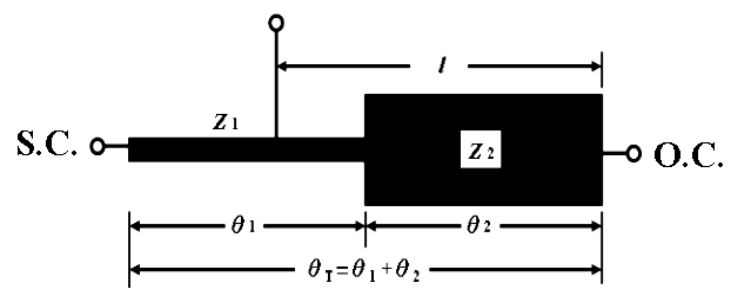

Fig. 1. Structure of a $\lambda / 4$ SIR.

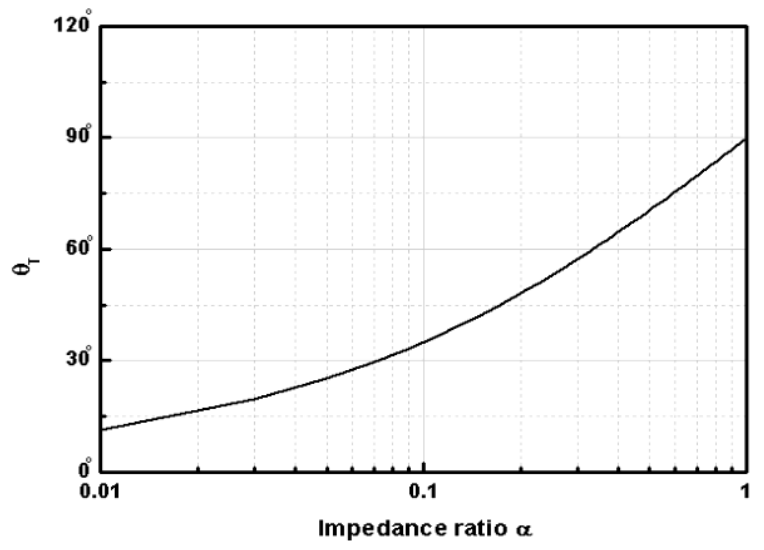

Fig. 2. SIR length versus impedance ratio in optimized design.

\section{OPtimAl Design FOR STEPPED IMPEDANCE RESONATORS}

Figure 1 shows a typical shorted quarter-wavelength SIR, which is composed of two sections of low-impedance and high-impedance transmission lines. The resonance condition of the $\lambda / 4$ SIR can be described by

$$
\tan \theta_{1}=\alpha \cdot \cot \theta_{2}
$$

where $\alpha$ is the impedance ratio of low-impedance and highimpedance defined as

$$
\alpha=\frac{Z_{2}}{Z_{1}}
$$

Herein, the fundamental resonance and the spurious resonances can be adjusted by choosing a suitable impedance 
ratio of the $\lambda / 4 \mathrm{SIR}$. As the impedance ratio of the $\lambda / 4 \mathrm{SIR}$ is kept smaller than unity, the fundamental resonance and the first resonance shifts to the lower and higher frequency values respectively, thus extending a very wide stopband.

In order to reduce the circuit size of the filter, the equation for the minimum length of the $\lambda / 4$ SIR can be derived as

$$
\theta_{1}=\theta_{2}=\tan ^{-1} \sqrt{\alpha}
$$

Fig. 2 plots the impedance ratio of $\lambda / 4$ SIR with respect to the SIR length $\theta_{\mathrm{T}}$, when $\theta_{1}=\theta_{2}$. It can be seen that the case for $\alpha=1$ stands for a traditional $\lambda / 4$ grounded unit impedance resonator (UIR), whose first spurious passband is centered at about three-times the mid-band frequency. As long as $\alpha$ is kept smaller than 1 , the length of $\lambda / 4$ SIR can be reduced when compared with that of the UIR.

\section{FiLTER DeSIGN: EXAMPLE ONE}

The first filter design example is the structure shown in Fig. 3 . In this example, the characteristic impedances of the highand low-impedance lines are chosen as $Z_{1}=50 \Omega$ and $Z_{2}=25 \Omega$ and it results in $\alpha=0.5$. Therefore, the length of the SIR is about one-fifth of guided wavelength as presented in Fig. 2. In the design process, each SIR is folded to achieve the cross coupling and to be compact, so that each SIR has the lateral size of only about one-fifteenth of guided wavelength. The four-pole cross-coupled filter was designed with the given specifications. The center frequency of the filter is $1 \mathrm{GHz}$, and the fractional bandwidth is $5.8 \%$. The coupling matrix $[\mathrm{m}]$ and the external quality factor $Q_{e}$ can then be found to be:

$$
\begin{aligned}
{[m] } & =\left[\begin{array}{cccc}
0 & 0.045 & 0 & -0.01 \\
0.045 & 0 & 0.046 & 0 \\
0 & 0.046 & 0 & 0.045 \\
-0.01 & 0 & 0.045 & 0
\end{array}\right] \\
Q_{e} & =15.83
\end{aligned}
$$

Full-wave simulator IE3D has been used to extract above parameters in order to obtain the physic dimensions of the filter. The filter design is based on the knowledge of the coupling coefficients of the three basic coupling structures as the electric, magnetic, and mixed couplings. The coupling coefficient is evaluated from two dominant resonant frequencies $f_{1}$ and $f_{2}$ given as [1]:

$$
M_{i j}=\frac{f_{2}^{2}-f_{1}^{2}}{f_{2}^{2}+f_{1}^{2}}
$$

where $M_{i j}$ represents the coupling coefficient between resonators $i$ and $j$. The external quality factor can be characterized by:

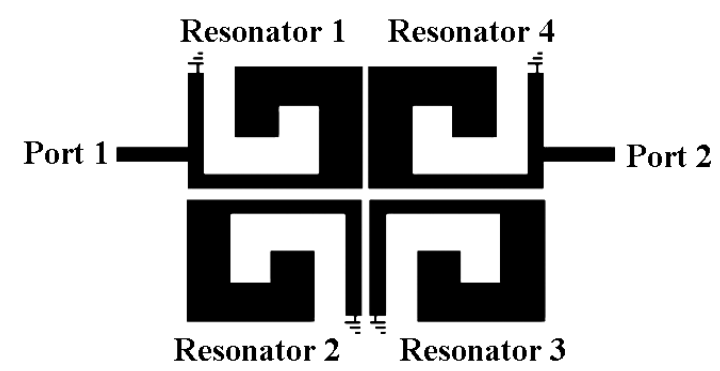

Fig. 3. Proposed structure of four-pole microstrip cross-coupled bandpass filter.

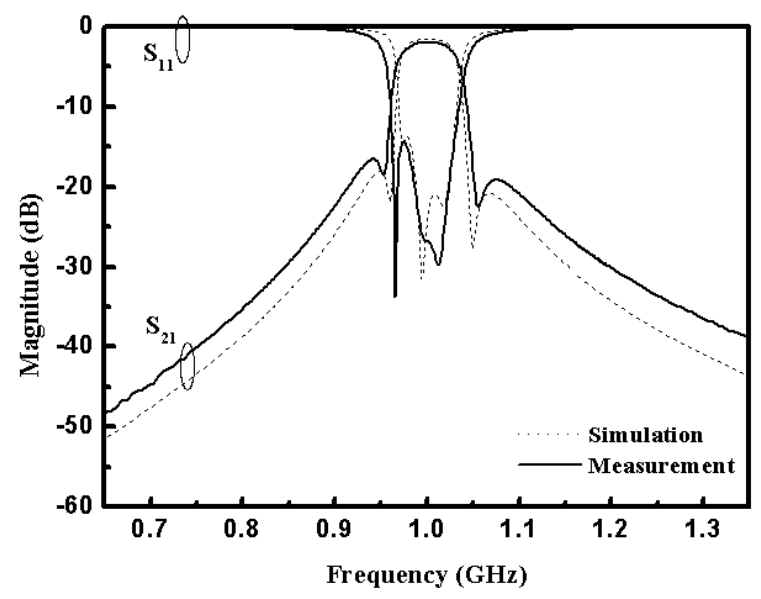

Fig. 4. Simulated and measured results of the first filter design.

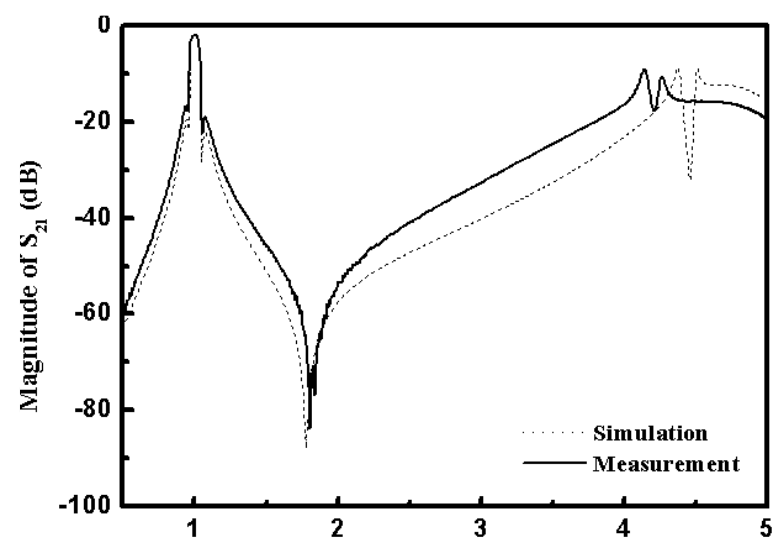

Fig. 5. Simulated and measured wide-band frequency responses of the first filter design.

$$
Q_{e}=\frac{f_{0}}{\delta f_{3-d B}}
$$

where $f_{0}$ and $\delta f_{3-\mathrm{dB}}$ represent the resonant frequency and the 3-dB bandwidth of the input or output resonator. 
The four-pole filter is designed to be fabricated using copper metallization on the Rogers RO4003 substrate with a relative dielectric constant of 3.38 , a thickness of $0.508 \mathrm{~mm}$, and a loss tangent of 0.0027 . The overall size of the filter is $25.1 \mathrm{~mm}$ by $19.9 \mathrm{~mm}$, i.e., only about $0.13 \lambda_{\mathrm{g}}$ by $0.11 \lambda_{\mathrm{g}}$, where $\lambda_{\mathrm{g}}$ is the guided wavelength of $50 \mathrm{ohm}$ line on the substrate at the center frequency. The fabricated filter was measured with an Agilent E5071B network analyzer. The measured and simulated results of this filter are shown in Fig. 4. The passband return loss is below $15 \mathrm{~dB}$ and the passband insertion loss is about $1.9 \mathrm{~dB}$. The insertion loss would mainly be attributed to the conductor loss. It can be clearly observed that the cross coupling effect produces a single pair of transmission zeros near the passband, which results in a higher selectivity.

Figure 5 shows the measured and simulated wide-band responses. It seems that the filter has a very wide stopband between the fundamental response and the first spurious response due to the choice of $\alpha<1$ in SIR. The stopband rejection is better than $20 \mathrm{~dB}$ from $1.05 \sim 3.8 \mathrm{GHz}$. It is also interesting to point out an extra transmission zero in the stopband between the fundamental response and the first spurious response, which occurs at the frequency when the length $l$ of the input resonator approximates a quarter guided wavelength, and it can be tuned by proper feeding at the first and the last resonators without increasing the circuit area when high attenuation may be needed only at certain frequencies.

\section{Filter Design: EXAmple Two}

Figure 6 is the structure of the designed filter. In this case, the characteristic impedances of $Z_{1}$ and $Z_{2}$ are further chosen to be $50 \Omega$ and $12.5 \Omega$, respectively, which give $\alpha=0.25$ for this example. The length of the SIR can then be obtained as about one-seventh of guided wavelength. Similarly, each SIR is folded to achieve the cross coupling and to be compact. Thus, it can be seem that the lateral physical length of the folded SIR is about one-fifteenth of guided wavelength. The bandpass filter is designed with the following specifications. The center frequency of the filter is $0.98 \mathrm{GHz}$, and the fractional bandwidth is $4.8 \%$. The coupling matrix $[\mathrm{m}]$ and the external quality factor $Q_{e}$ of the filter are found to be:

$$
\begin{aligned}
{[m] } & =\left[\begin{array}{cccc}
0 & 0.042 & 0 & -0.014 \\
0.042 & 0 & 0.035 & 0 \\
0 & 0.035 & 0 & 0.042 \\
-0.014 & 0 & 0.042 & 0
\end{array}\right] \\
Q_{e} & =16.86
\end{aligned}
$$

The filter is also fabricated on the Rogers RO4003 substrate. The size of the filter amounts to $20.8 \mathrm{~mm}$ by $25.2 \mathrm{~mm}$, i.e., only about $0.11 \lambda_{\mathrm{g}}$ by $0.13 \lambda_{\mathrm{g}}$. The simulated and measured results are shown in Fig. 7. The passband insertion loss is about $2.9 \mathrm{~dB}$, which is mainly attributed to the

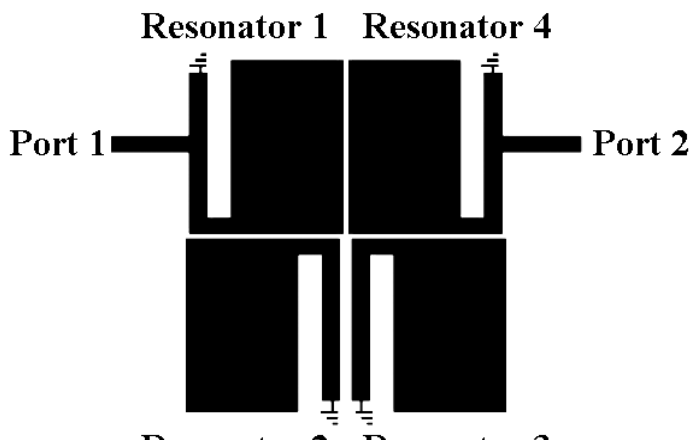

Resonator 2 Resonator 3

Fig. 6. Proposed structure of four-pole microstrip cross-coupled bandpass filter.

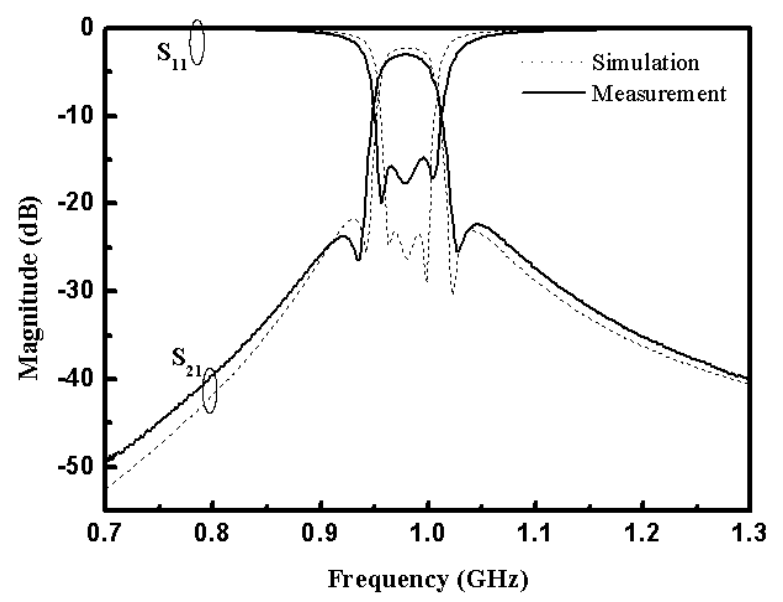

Fig. 7. Simulated and measured results of the second filter design.

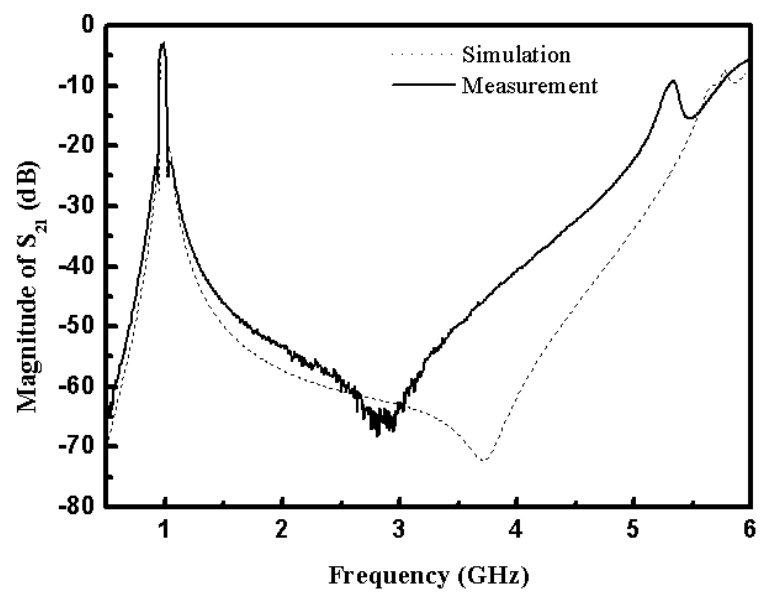

Fig. 8. Simulated and measured wide-band frequency responses of the second filter design.

conductor loss. The passband return loss is greater than $15 \mathrm{~dB}$.

Figure 8 shows the measured and simulated wide-band responses. It is also found that the filter has a very wide 
stopband of up to about $5.8 f_{0}$, which is wider than that for the previous design example. The stopband rejection is better than $20 \mathrm{~dB}$ from $1.02 \sim 5.1 \mathrm{GHz}$. Again, by adjusting the feed point of input/output SIR, the extra transmission zero in the upper stopband can be tuned in order to enhance the selectivity of the stopband. As a result, the compact miniaturized bandpass filter not only has a high selectivity, but also has a very wide stopand between the fundamental response and the first spurious response.

\section{CONCLUSION}

Novel compact microstrip cross-coupled bandpass filters using miniaturized SIR's have been proposed in this study. The cross coupling effect which created two transmission zeros to the filter response is realized in order to improve the selectivity of the filter. By employing the optimal design for miniaturized quarter-wavelength SIR, the compact filter size can then be obtained. The miniaturized SIR is presented not only to reduce the filter size, but also to control the spurious-mode frequencies, which has been verified by the above two experimental results. In addition, an extra transmission zero occurs at the upper stopband, which is caused by the feeding structure and can be tuned by proper feeding at the first and the last resonators for better rejection of interference signal. As a result, the proposed filters, which have advantages of small size, high-selectivity, and wide stopband response, are quite useful for future applications in wireless communication systems.

\section{ACKNOWLEDGEMENT}

This work was supported in part by the National Science Council, Taiwan, R.O.C. under Grant NSC 93-2752-E-002003-PAE.

\section{REFERENCES}

[1] J. S. Hong and M. J. Lancaster, "Couplings of microstrip square openloop resonators for cross-coupled planar microwave filters," IEEE Trans. Microwave Theory Tech., MTT-44, pp. 2099-2109, Nov. 1996.

[2] J. S. Hong and M. J. Lancaster, "Theory and experiment of novel microstrip slow-wave open-loop resonator filters," IEEE Trans. Microwave Theory Tech., vol. 45, pp. 2358-2365, Dec. 1997.

[3] J. S. Hong and M. J. Lancaster, "Design of highly selective microstrip bandpass filters with a single pair of attenuation poles at finite frequencies," IEEE Trans. Microwave Theory Tech., vol. 48, pp. 1098-1107, July. 2000.

[4] J. S. Hong and M. J. Lancaster, "Cross-coupled microstrip hairpinresonator filters," IEEE Trans. Microwave Theory Tech., vol. 46, pp. 118-122, Jan. 1998.

[5] J. T. Kuo, M. J. Maa, and P. H. Lu, "A microstrip elliptic function filter with compact miniaturized hairpin resonators," IEEE Trans. Microwave Guided Wave Lett., vol. 10. pp. 94-95, Sept. 2000.

[6] S. Y. Lee and C. M. Tsai, "New cross-coupled filter design using improved hairpin resonators," IEEE Trans. Microwave Theory Tech., vol.48, pp. 2482-2490, Dec. 2000.
[7] K. S. Yeo and M.J. Lancaster, "The design of microstrip six-pole quasi-elliptic filter with linear phase response using extracted-pole technique," IEEE Trans. Microwave Theory Tech., vol.49, pp. 321-327, Feb. 2001.

[8] C. C. Chen, Y. R. Chen, and C. Y. Chang, "Miniaturized microstrip cross-coupled filters using quarter-wave or quasi-quarter-wave resonators," IEEE Trans. Microwave Theory Tech., vol. 51, pp. 120-131, Jan. 2003.

[9] S. B. Cohn, "Parallel coupled transmission-line resonator filters," IRE Trans. Microwave Theory Tech., vol. MTT-6, pp. 223-231, Apr. 1958.

[10] E. G. Cristal and S. Frankel, "Hairpin-line and hybrid hairpin-line/half-wave parallel-coipled-line filters," IEEE Trans. Microwave Theory Tech., vol. MTT-20, pp. 719-728, Nov. 1972.

[11] M. Makimoto and S. Yamashita, "Bandpass filters using parallel coupled stripline stepped impedance resonators," IEEE Trans. Microwave Theory Tech., vol.28, pp. 1413-1417, Dec. 1980.

[12] M. Sagawa, M. Makimoto, and S. Yamashita, "Geometrical structures and fundamental characteristics of microwave stepped-impedance resonators," IEEE Trans. Microwave Theory Tech., vol.45, pp. 1078-1085, July 1997.

[13] J. T. Kuo and E. Shih, "Microstrip stepped impedance resonator bandpass filter with an extended optimal rejection bandwidth," IEEE Trans. Microwave Theory Tech., vol.51, pp. 1554-1559, May 2003. 